\title{
Towards Accurate Text-based Image Captioning with Content Diversity Exploration
}

\author{
Guanghui $\mathrm{Xu}^{1,2 *}$, Shuaicheng Niu ${ }^{1 *}$, Mingkui Tan ${ }^{1,4}$, Yucheng $\mathrm{Luo}^{1}$, Qing Du ${ }^{1,4 \dagger}, \mathrm{Qi} \mathrm{Wu}^{3}$ \\ ${ }^{1}$ South China University of Technology, ${ }^{2}$ Pazhou Laboratory, ${ }^{3}$ University of Adelaide \\ ${ }^{4}$ Key Laboratory of Big Data and Intelligent Robot, Ministry of Education, \\ sexuguanghui@mail.scut.edu.cn, \{mingkuitan, duqing\}@scut.edu.cn, qi.wu01@adelaide.edu.au
}

\begin{abstract}
Text-based image captioning (TextCap) which aims to read and reason images with texts is crucial for a machine to understand a detailed and complex scene environment, considering that texts are omnipresent in daily life. This task, however, is very challenging because an image often contains complex texts and visual information that is hard to be described comprehensively. Existing methods attempt to extend the traditional image captioning methods to solve this task, which focus on describing the overall scene of images by one global caption. This is infeasible because the complex text and visual information cannot be described well within one caption. To resolve this difficulty, we seek to generate multiple captions that accurately describe different parts of an image in detail. To achieve this purpose, there are three key challenges: 1) it is hard to decide which parts of the texts of images to copy or paraphrase; 2) it is non-trivial to capture the complex relationship between diverse texts in an image; 3) how to generate multiple captions with diverse content is still an open problem. To conquer these, we propose a novel Anchor-Captioner method. Specifically, we first find the important tokens which are supposed to be paid more attention to and consider them as anchors. Then, for each chosen anchor, we group its relevant texts to construct the corresponding anchor-centred graph (ACG). Last, based on different ACGs, we conduct the multi-view caption generation to improve the content diversity of generated captions. Experimental results show that our method not only achieves SOTA performance but also generates diverse captions to describe images.
\end{abstract}

\section{Introduction}

The texts are omnipresent in our daily life and play an important role in helping humans or intelligent robots to

\footnotetext{
*Authors contributed equally.

${ }^{\dagger}$ Corresponding author
}

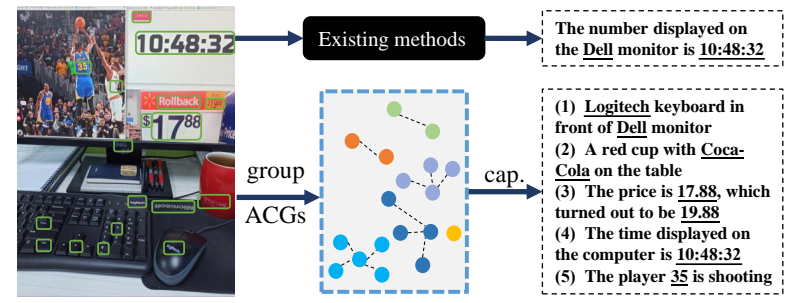

Figure 1. Comparison with existing methods. For a given image, existing methods tend to generate only one global caption. Unlike them, we first select and group texts to anchor-centred graphs (ACGs), and then decide which parts of the texts to copy or paraphrase. Our method is able to achieve higher accuracy and generate diverse captions to describe the image from different views.

understand the physical world [13]. In the image captioning area, the texts contained in images are also of critical importance and often provide valuable information $[5,19,20,34,41]$ for caption generation. In this sense, Sidorov et al. [40] propose a fine-grained image captioning task, i.e., text-based image captioning (TextCap), aiming to generate image captions that not only 'describe' visual contents but also 'read' the texts in images, such as billboards, road signs, commodity prices and etc. This task is very practical since the fine-grained image captions with rich text information can aid visually impaired people to comprehensively understand their surroundings [13]

Some preliminary tries for the TextCap task seek to directly extend existing image captioning methods [2, 19, 21] to this new setting. However, such methods usually tend to describe prominent visual objects or overall scenes without considering the texts in images. Recently, M4CCaptioner [40] tries to use additional OCR tools [4, 6, 31] to recognise texts in images. It is still hard to well describe the complex text and visual information within one caption. To resolve this difficulty, we propose to generate multiple diverse captions focusing on describing different parts of an image. However, there are still some challenges.

First, it is hard to decide which parts of the texts are most 
crucial for describing the images. As shown in Figure 1, an image often contains a lot of texts, but only a small part of the texts play a key role in caption generation. For example, a PC keyboard contains many letters, but we do not need a caption that covers all the recognised letters.

Second, it is non-trivial to capture the complex relationship between diverse texts in an image. The correct understanding of such a relationship is essential for generating accurate captions. For example, to accurately describe a cup, we might use its brand and capacity. But these texts have no relevance to the contents on the computer screen.

More critically, how to generate multiple captions describing different contents remains unknown. Current image captioning methods $[2,19,21]$ often only generate a content-monotone caption. They tend to focus on a small part of the contents in the image, such as the time in the monitor in Figure 1. To comprehensively describe an image, one better solution is to generate diverse captions, where each caption focuses on describing one relevant part.

To address the above issues, we design a new AnchorCaptioner architecture that mainly consists of two key modules, i.e., an anchor proposal module (AnPM) and an anchor captioning module (AnCM). Specifically, AnPM is proposed to understand the texts in an image and to capture the complex relationships between different texts. To be specific, we first employ an anchor predictor to rank the importance of each token. Then, we choose several important tokens to decide which parts of texts are most informative and need to be carefully considered. After that, considering each chosen token as an anchor, we use a recurrent neural network to model its complex relationships with other tokens and to construct an anchor-centred graph (ACG) for each anchor. Each ACG denotes a group of the relevant tokens which are supposed to be included in the same caption. Based on the different ACGs for an image, we apply AnCM to generate diverse captions that cover various OCR tokens. To be specific, we first generate a visualspecific caption to model global visual information. Then, we take each ACG as guidance to refine the visual caption and generate multiple text-specific captions that contain fine-grained text information. Extensive experimental results on TextCaps dataset demonstrate the effectiveness of our proposed method.

In summary, our main contributions are as follows:

1. We propose to exploit fine-grained texts information to generate multiple captions that describe different parts of images, instead of generating a single caption to handle them as a whole.

2. We propose an anchor proposal module (AnPM) and an anchor captioning module (AnCM) to select and group texts to anchor-centred graphs (ACGs) and then generate diverse captions based on ACGs.
3. We achieve the state-of-the-art results on TextCaps dataset, in terms of both accuracy and diversity.

\section{Related work}

Image captioning aims to automatically generate textual descriptions of an image, which is an important and complex problem since it combines two major artificial intelligence fields: natural language processing and computer vision. Most image captioning models [2, 15, 42, 44, 45, 49] use CNNs to encode visual features and apply RNNs as language decoder to generate descriptions. Some works [16, $25,29,48]$ propose to further refine the generated sentences with multiple decoding passes. NBT [32] first generates a template without specifics and then fills it with 'object' words. RL-based methods [18, 30, 35, 39] model the sequence generation as Markov Decision Process [47] and directly maximise the metric scores.

To generate diverse image captions, many works try to control the generation in terms of style and contents. The style controllable methods [14, 17, 33] usually require additional annotations for training, such as a pair of labelled captions with different styles. Other parallel works focus on controlling the contents of the generated captions. Johnson et al. [22] are the first to propose the dense captioning task to describe the visual objects in a sub-region [50]. Signal-based methods [7, 8, 9, 11] sample different predictions based on the control signals to obtain diverse image captions. Our work can be seen as text-based dense captioning and aims to generate multi-view captions.

Text-based image captioning aims to generate captions describing both the visual objects and written texts. Intuitively, the text information is important for us to understand the image contents. However, the existing image captioning datasets $[24,28]$ have a bias that only describes the salient visual objects in the image while ignoring the text information. As a result, most image captioning models [2, 15, 42, 44, 49] unable to 'read' the texts since they don't pay attention to improve such ability. In this sense, Sidorov et al. [40] introduce a novel dataset, namely TextCaps, which requires a captioning model not only to 'watch' visual contents but also 'read' the texts in images. They introduce a benchmark M4C-Captioner [40], which is simply extended from the M4C [19] (for TextVQA). Specifically, they feed all the detected texts and visual contents into their captioning model to generate a global caption for an input image. However, it is difficult for a single caption to cover all the multimodal information, and the overlooked parts may be the information that people are interested in.

Different from existing methods, we propose an anchor proposal module to understand the relationship within OCR tokens and group them to construct anchor-centred graphs (ACGs). With the help of ACGs, our method is able to better describe the input image by generating diverse captions. 


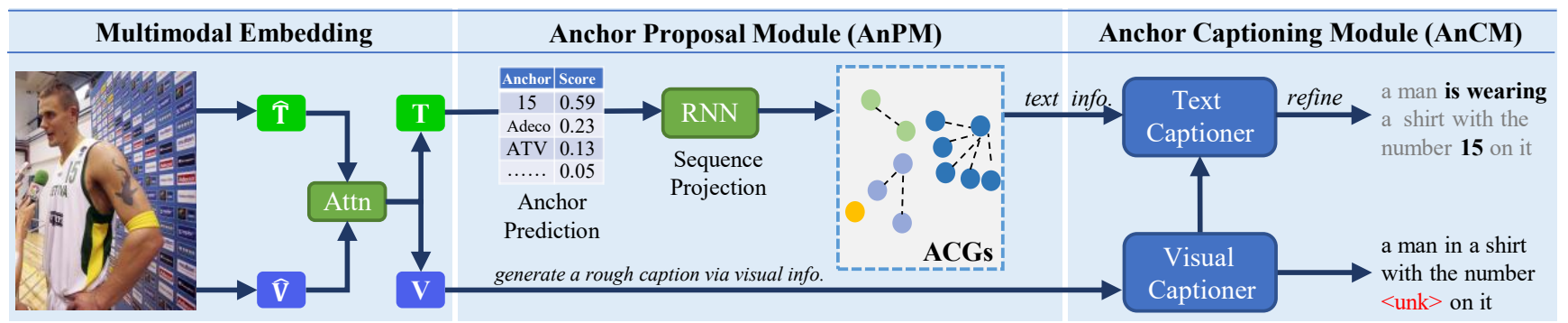

Figure 2. An illustration of Anchor-Captioner. Given an input image, (1) we first extract text and visual features $(\widehat{\mathbf{T}}, \widehat{\mathbf{V}})$ independently. Then, we fuse them to obtain multimodal features (T, V) via self-attention; (2) Following that, AnPM chooses a series of anchor-tokens based on the anchor predictions and then groups the relevant tokens by constructing anchor-centred graphs (ACGs). (3) Lastly, AnCM employs a visual-captioner to output a global visual-specific caption, and then uses a text-captioner to generate multiple text-specific captions based on the above global caption and ACGs. In this figure, we only show the generated caption with the ACG of the top-1 score.

\section{Proposed method}

We study text-based image captioning (TextCap) which aims to read and reason an image with texts to generate detailed captions. This task is very challenging because it is difficult to comprehensively describe images with rich information. Existing methods tend to generate one global caption that tries to describe complex contents in an image. However, such methods are unfeasible when the image contains a large number of redundant visual objects and diverse texts. To accurately describe an image, one better solution is to generate multiple captions from different views. However, several challenges still exist. First, it is hard to decide which parts of the texts of images to copy or paraphrase when images contain a lot of texts. Second, it is non-trivial to exploit the correct relationship between diverse texts in an image, which, however, is essential to accurately describe the image. More critically, how to generate multiple captions from different views for comprehensively describing images is still unknown.

In this paper, we propose a new captioning method Anchor-Captioner that aims to accurately describe images by using content diversity exploration. As shown in Figure 2, Anchor-Captioner has two main components, i.e., an anchor proposal module (AnPM) and an anchor captioning module (AnCM). AnPM chooses important texts as anchors and constructs anchor-centred graphs (ACGs) to model complex relationship between texts. AnCM takes different ACGs as input to generate multiple captions that describe different parts of an image. In this way, our method is able to choose important relevant texts to describe and also has the ability to generate diverse captions for comprehensively understanding images.

\subsection{Multimodal embedding}

To generate captions for an image, we first use a pretrained Faster RCNN [38] model to extract $N$ visual objects and recognise $M$ OCR tokens by the Rosetta OCR [6]. Visual embedding. For the $i$-th visual object, the Faster
RCNN model outputs appearance feature $\mathbf{v}_{i}^{a} \in \mathbb{R}^{d}$ and a 4-dimensional bounding box coordinate $\mathbf{v}_{i}^{b}$. To enrich the visual representation, we apply a linear layer $f_{1}$ with LayerNorm [3] to project the above features as $\widehat{\mathbf{v}}_{i}=f_{1}\left(\left[\mathbf{v}_{i}^{a}, \mathbf{v}_{i}^{b}\right]\right)$, where $[\cdot, \cdot]$ is a concatenation operation.

Token embedding. For each recognised OCR token, we also use its appearance feature $\mathbf{t}_{i}^{a}$ and bounding box coordinate $\mathbf{t}_{i}^{b}$. Apart from these features, following the M4CCaptioner [40], we adopt two additional text features to further enrich the representations, including FastText feature $\mathbf{t}_{i}^{f}$ and PHOC (pyramidal histogram of characters) feature $\mathbf{t}_{i}^{p}$. In particular, $\mathbf{t}_{i}^{f}$ is a pretrained word-level embedding for written texts while $\mathbf{t}_{i}^{p}$ is a character-level embedding for capturing what characters are present in the tokens. Based on the rich representations of OCR tokens, we calculate OCR token features by $\widehat{\mathbf{t}}_{i}=f_{2}\left(\left[\mathbf{t}_{i}^{a}, \mathbf{t}_{i}^{b}, \mathbf{t}_{i}^{f}, \mathbf{t}_{i}^{p}\right]\right)$, where $f_{2}$ is a linear layer with LayerNorm to ensure that token embedding has the same scale as visual embedding.

Multimodal embedding fusion. Based on the above, we obtain visual embedding $\widehat{\mathbf{V}}=\left[\widehat{\mathbf{v}}_{1}, \ldots, \widehat{\mathbf{v}}_{N}\right]^{\top}$ and token embedding $\widehat{\mathbf{T}}=\left[\widehat{\mathbf{t}}_{1}, \ldots, \widehat{\mathbf{t}}_{M}\right]^{\top}$. Since both the OCR tokens and visual objects are visual contents and exist in images as a whole, it is necessary to model their interaction. Formally, given $\widehat{\mathbf{V}}$ and $\widehat{\mathbf{T}}$, we use an $L_{1}$-layer Transformer module $\Psi\left(\cdot ; \theta_{a}\right)$ to obtain more informative features via a self-attention operation as

$$
\mathbf{V}, \mathbf{T}=\Psi\left([\widehat{\mathbf{V}}, \widehat{\mathbf{T}}] ; \theta_{a}\right) .
$$

\subsection{Anchor proposal module}

Based on the multimodal embeddings $(\mathbf{V}, \mathbf{T})$, existing TextCap methods such as M4C-Captioner [40] simply treat the texts in an image as another kind of visual information and feed them to a captioning module without distinction. However, compared with ambiguous visual information, the texts in images are essential to describe images and thus need to be considered carefully.

To this end, we propose the anchor proposal module (AnPM) to determine which OCR tokens should be paid 
more attention to describe. Inspired by the region proposal network (RPN), AnPM first performs anchor prediction among OCR tokens to output a score for each token and choose a series of important tokens as anchors. After that, to model the complex relationship between tokens, AnPM groups relevant tokens to construct the corresponding anchor-centred graph (ACG) for each anchor. Now, we introduce how to construct ACGs in detail.

Anchor prediction. Intuitively, different OCR tokens play different roles in caption generation. However, it is hard to decide which texts should be paid more attention to. To this end, based on the text features $\mathbf{T}$, we apply a linear layer $\phi$ as an anchor predictor to predict a score for each token as

$$
\mathbf{s}_{\text {anchor }}=\operatorname{Softmax}(\phi(\mathbf{T})) .
$$

In the anchor score $\mathbf{s}_{\text {anchor }} \in \mathbb{R}^{M}$, each element indicates the importance weight of each OCR token. In training, we adopt the OCR token with the highest score as the anchor by argmax operation, denoted as

$$
\mathbf{T}_{\text {anchor }}=\mathbf{T}_{i,:}, \quad \text { where } i=\operatorname{argmax}\left(\mathbf{s}_{\text {anchor }}\right) .
$$

After that, we can obtain the anchor embedding $\mathbf{T}_{\text {anchor }}$. During the inference phase, we choose OCR tokens with top- $K$ scores as anchors.

Anchor-centred graph construction. In this paper, we employ a RNN module and take $\mathbf{T}_{\text {anchor }}$ as the initial hidden state to model the potential dependence between the anchor and different tokens. The ACG construction for the anchor $\mathbf{T}_{\text {anchor }}$ can be formulated as:

$$
\begin{aligned}
\mathbf{T}_{\text {graph }} & =\mathrm{RNN}\left(\mathbf{T}, \mathbf{T}_{\text {anchor }}\right), \\
\mathbf{s}_{\text {graph }} & =\sigma\left(f_{3}\left(\mathbf{T}_{\text {graph }}\right)\right),
\end{aligned}
$$

where $\mathbf{T}_{\text {graph }} \in \mathbb{R}^{M \times d}$ denotes the updated token feature and $f_{3}$ is a linear layer followed by Sigmoid activation function $\sigma$ to output the graph score $\mathbf{s}_{\text {graph }} \in \mathbb{R}^{M}$ for all $M$ tokens. After that, we concatenate $\mathbf{T}_{\text {anchor }}$ and its relevant tokens to construct the ACG $\mathcal{G}$ as follows:

$$
\begin{gathered}
\mathcal{G}=\left[\mathbf{T}_{\text {anchor }},\left\{\mathbf{T}_{\text {graph }}^{i}\right\}\right], \quad \text { where } \\
\mathbf{s}_{\text {graph }}^{i}>0.5, \forall i \in[1, M] .
\end{gathered}
$$

Overall, the anchor proposal module (AnPM) learns to select an important OCR token as an anchor and then construct an ACG for it. In this way, AnPM is able to propose a series of ACGs for an input image, which would be fed into the captioning module as guidance to generate diverse captions. Meanwhile, the generation process of each ACG is independent and will not be affected by other pairs, which greatly improves the quality of the generated captions.

\subsection{Anchor captioning module}

Compared with general image captioning, the TextCap requires captioning models to not only describe visual objects but also contain OCR tokens in the generated captions. To achieve this, we carefully design a progressive captioning module, namely Anchor Captioning Module (AnCM). Inspired by the Deliberation Network [48], as shown in Figure 2, AnCM consists of a visual-captioner (denoted as $\mathrm{AnCM}_{v}$ ) and a text-captioner (denoted as $\mathrm{AnCM}_{t}$ ). First, the visual-captioner, a standard image captioning module, uses the updated visual embedding $\mathbf{V}$ to generate a visual-specific caption $\mathcal{Y}^{\prime}$ with $C$ words, denoted as $\mathcal{Y}^{\prime}=$ $\left\{y_{c}^{\prime}\right\}_{c=1}^{C}$. Then, the text-captioner is proposed to refine the generated caption based on the text information of ACG $\mathcal{G}$ (see Eqn. (5)). Following the training of sequence generation task, the goal of AnCM is to maximise the data log likelihood function as follows:

$$
\log \sum_{c=1}^{C} P\left(y_{c} \mid \operatorname{AnCM}_{t}\left(y_{c}^{\prime}, \mathcal{G}\right)\right) P\left(y_{c}^{\prime} \mid \operatorname{AnCM}_{v}(\mathbf{V})\right),
$$

where $\left\{y_{c}\right\}$ is the final generated caption. Since the predicted token $y_{c}^{\prime}$ is obtained by argmax function which is a non-differentiable operation, we cannot directly optimise the above equation. To address this issue, we feed the hidden state feature $\mathbf{h}_{c}$ outputted from $\mathrm{AnCM}_{v}$ directly into $\mathrm{AnCM}_{t}$ and the training loss for $\mathrm{AnCM}_{t}$ is computed as follows:

$$
\begin{gathered}
\mathcal{L}_{\text {tcap }}=-\log \sum_{c=1}^{C} P\left(y_{c} \mid \operatorname{AnCM}_{t}\left(\mathbf{h}_{c}, \mathcal{G}\right) ; \theta_{t}\right), \text { where } \\
\mathbf{h}_{c}=\operatorname{AnCM}_{v}\left(\mathbf{V} ; \theta_{v}\right) .
\end{gathered}
$$

The $\theta_{v}$ and $\theta_{t}$ are learnable parameters of visual-captioner and text-captioner, respectively. In this way, we can train AnCM in an end-to-end manner. Next, we will introduce more details about the two captioners.

Visual-captioner $\left(\mathrm{AnCM}_{v}\right)$. To capture long-range dependency in sequence modelling, we employ an $L_{2}$-layer Transformer module $(\Psi)$ as the backbone of the visualcaptioner. Specifically, the visual-captioner generates tokens in an auto-regressive manner as follows:

$$
\begin{aligned}
\mathbf{h}_{c} & =\Psi\left(\mathbf{V}, \operatorname{LM}\left(\mathbf{y}_{c-1}^{\prime}\right) ; \theta_{v}\right), \\
y_{c}^{\prime} & =\operatorname{argmax}\left(f_{4}\left(\mathbf{h}_{c}\right)\right),
\end{aligned}
$$

where $\mathbf{y}_{c-1}^{\prime}$ denotes the embedding of previous output token, $f_{4}(\cdot)$ is a linear classifier for common vocabulary and we can obtain the predicted token $y_{c}^{\prime}$ with argmax operation. Here, we use the prefix language modelling (LM) technique [37] to ensure that the input entries only use previous predictions, and avoid peeping at subsequent generation processes. Thus far, with the help of the visual-captioner, 
we obtain a visual-specific caption $\left\{y_{c}^{\prime}\right\}$ and its hidden state feature $\left\{\mathbf{h}_{c}\right\}_{c=1}^{C}$. Formally, we define the training loss for $\mathrm{AnCM}_{v}$ as $\mathcal{L}_{v c a p}=-\log \sum_{c=1}^{C} P\left(y_{c}^{\prime}\right)$.

Image captioning is a fairly mature sequence-generation task, and researchers have also proposed many models with promising performance. In this work, we do not focus on designing a new captioning module. Intuitively, the backbone of visual-captioner can be easily replaced by other image captioning models, such as BUTD [2] and AoANet [21].

Text-captioner $\left(\mathrm{AnCM}_{t}\right)$. Based on the hidden state features $\left\{\mathbf{h}_{c}\right\}_{c=1}^{C}$, the text-captioner aims to generate textspecific captions that contain given OCR tokens. To this end, at this stage, we use ACG as the guidance to refine the caption generated by the visual-captioner from the last step. Specifically, we use an $L_{3}$-layer Transformer module $(\Psi)$ as the backbone of the text-captioner. Relying on self-attention, the Transformer allows multimodal embedding to freely interact with others, thereby achieving satisfactory progress in sequence generation tasks. Formally, given the hidden state and ACGs, our $\mathrm{AnCM}_{t}$ can output the joint embedding as follows:

$$
\widehat{\mathcal{G}}, \widehat{\mathbf{y}}_{c}=\Psi\left(\left[\mathcal{G}, \mathbf{h}_{c}, \operatorname{LM}\left(\mathbf{y}_{c-1}\right)\right] ; \theta_{t}\right),
$$

where $\widehat{\mathcal{G}}$ is the updated token embedding in the ACG and $\widehat{\mathbf{y}}_{c}$ is the embedding of $c$-th prediction. Following M4C [19], we adopt different classifiers for common vocabulary and OCR candidate tokens as

$$
y_{c}=\operatorname{argmax}\left(\left[f_{4}\left(\widehat{\mathbf{y}}_{c}\right), f_{d p}\left(\widehat{\mathcal{G}}, \widehat{\mathbf{y}}_{c}\right)\right]\right),
$$

where $f_{4}$ is the shared classifier with visual-captioner in Eqn. (8), $f_{d p}$ denotes the dynamic pointer network [19] that makes prediction based on the $\widehat{\mathcal{G}}$ and $\widehat{\mathbf{y}}_{c}$. After concatenating two prediction scores, we use the argmax function on the final prediction score to obtain the predicted token $y_{c}$.

Compared with general captioning methods, the proposed AnCM makes full use of a key character of the TextCap task, that is, the OCR token can be used as an important guide to improve the generation accuracy.

\subsection{Training details}

Formally, we train our Anchor-Captioner model by optimising the following loss function:

$$
\begin{aligned}
\mathcal{L}= & \mathcal{L}_{\text {anchor }}\left(\mathbf{s}_{\text {anchor }}\right)+\alpha \mathcal{L}_{\text {graph }}\left(\mathbf{s}_{\text {graph }}\right) \\
& +\beta \mathcal{L}_{\text {vcap }}\left(\mathcal{Y}^{\prime}\right)+\eta \mathcal{L}_{\text {tcap }}(\mathcal{Y})
\end{aligned}
$$

where the $\mathbf{s}_{\text {anchor/graph }}$ is the output of AnPM (see Eqn. (2) or (4)), the $\mathcal{Y}^{\prime}=\left\{y_{c}^{\prime}\right\}$ and $\mathcal{Y}=\left\{y_{c}\right\}$ denote the generated visual-specific caption and text-specific caption (see Eqns. (8) and (10)), respectively. $\{\alpha, \beta, \gamma\}$ are trade-off parameters. In practice, all the above four losses adopt the binary cross-entropy loss function. We train AnPM with $\mathcal{L}_{\text {anchor }}$ and $\mathcal{L}_{\text {graph }}$ to find the most frequently described ACGs. We train AnCM with $\mathcal{L}_{\text {vcap }}$ and $\mathcal{L}_{\text {tcap }}$ to generate visual-specific and text-specific captions. Due to the page limitation, we put detailed training and inference algorithms in Supplementary A.

Ground-truth labels. Next, we will illustrate how to obtain supervisions for training AnPM and AnCM. 1) Given a manually annotated caption (e.g.,'a man ... number $\underline{15}$ on $i t^{\prime}$ in Figure 2), we consider it as ground truth (gt) for $\underline{\mathcal{L}}_{\text {tcap }}$ to train the text-captioner. 2) We then mask the OCR tokens contained in the manually generated caption with [unk] to build a new caption (e.g.,' a man ... number [unk] on it') for $\mathcal{L}_{\text {vcap }}$ to train the visual-captioner. This is because we do not require the $\mathrm{AnCM}_{v}$ to make prediction among OCR tokens by using only the visual information. 3) Considering that different people will describe the same image from different views, the most frequently described token is often the most important one for describing an image. Thus, we choose the most frequently described token as gt for $\mathcal{L}_{\text {anchor }}$ to train the anchor prediction module in AnPM. 4) Given the chosen anchor, we consider the tokens that appear in the same caption as gt for $\mathcal{L}_{\text {graph }}$ to train AnPM to find the most relevant tokens for an anchor. During the test phase, we do not need to construct gt-ACGs since they are only used for calculating losses in the training. Note that the gt-ACGs are automatically mined and constructed from the same training split without using any additional annotations, and thus comparisons with other methods are fair.

\section{Experiments}

We verify the effectiveness of our method on the TextCaps [40] dataset. In the following, we first briefly introduce the TextCaps and the comparison settings for it in Sec. 4.1. More implementation details can be found in the Sec. 4.2. And then, we compare our method with existing captioning models in Sec. 4.3 and Sec. 4.4. Last, we demonstrate our proposed method by providing some visualisation results and analysis in Sec. 4.5.

\subsection{Datasets and settings}

Datasets. The TextCaps dataset [40] is collected from Open Image V3 dataset and contains 142,040 captions on 28,408 images, which have been verified to contain text through the Rosetta OCR system [6] and human annotators. For each image, there are five independent captions. In the test split, each image has an additional caption that is collected to estimate human performance on the dataset. The dataset also contains captions where OCR tokens are not presented directly but are used to infer a description [40]. In this case, the captioning models are required to perform challenging reasoning rather than simply copy the OCR tokens. Most 


\begin{tabular}{clccccc}
\hline \multirow{2}{*}{$\#$} & \multirow{2}{*}{ Method } & \multicolumn{5}{c}{ TextCaps validation set metrics } \\
\cline { 3 - 7 } & & $\mathrm{B}$ & $\mathrm{M}$ & $\mathrm{R}$ & $\mathrm{S}$ & $\mathrm{C}$ \\
\hline 1 & BUTD & 20.1 & 17.8 & 42.9 & 11.7 & 41.9 \\
2 & AoANet & 20.4 & 18.9 & 42.9 & 13.2 & 42.7 \\
3 & M4C-Captioner $^{-}$ & 23.3 & 22.0 & 46.2 & 15.6 & 89.6 \\
4 & M4C-Captioner $^{-}$ & 15.9 & 18 & 39.6 & 12.1 & 35.1 \\
5 & AnCM $_{v}$ & 16.1 & 16.3 & 40.1 & 11.2 & 29.1 \\
6 & Ours & $\mathbf{2 4 . 7}$ & $\mathbf{2 2 . 5}$ & $\mathbf{4 7 . 1}$ & $\mathbf{1 5 . 9}$ & $\mathbf{9 5 . 5}$ \\
\hline \multirow{2}{*}{$\#$} & \multirow{2}{*}{ Method } & TextCaps test set metrics \\
\cline { 3 - 7 } & & M & R & S & C \\
\hline 7 & BUTD & 14.9 & 15.2 & 39.9 & 8.8 & 33.8 \\
8 & AoANet & 15.9 & 16.6 & 40.4 & 10.5 & 34.6 \\
9 & M4C-Captioner & 18.9 & 19.8 & 43.2 & 12.8 & 81.0 \\
10 & MMA-SR & 19.8 & 20.6 & 44.0 & 13.2 & $\mathbf{8 8 . 0}$ \\
11 & Ours & $\mathbf{2 0 . 7}$ & $\mathbf{2 0 . 7}$ & $\mathbf{4 4 . 6}$ & $\mathbf{1 3 . 4}$ & 87.4 \\
\hline 12 & Human & 24.4 & 26.1 & 47.0 & 18.8 & 125.5 \\
\hline
\end{tabular}

Table 1. Comparison with SOTA methods on the validation and test set. In particular, rows 4 are captioning models without OCRs, i.e., only use visual information to generate captions. The last row is the estimated human performance, which can be seen as the upper bound of captioning models on the TextCaps dataset.

captions contain two or more OCR tokens, and the average length of captions is 12.4 .

Evaluation metrics. We use five standard evaluation metrics in image captioning, including BLEU (B) [36], METEOR (M) [10], ROUGE_L (R) [27], SPICE (S) [1] and CIDEr (C) [43] to evaluate the accuracy. Following the benchmark setting [40], we mainly focus on CIDEr, which puts more weight on informative tokens and is more suitable for this dataset. To evaluate the diversity of generated captions, we use Div-n [26] and SelfCIDEr [46] metrics on the validation set. In particular, the Div-n focuses on tokenlevel diversity while the SelfCIDEr is used for semanticlevel diversity. In addition, we propose a new metric, called Cover Ratio (CR), to measure the content diversity, that is, how many OCR tokens are included in the generated captions. For notation convenience, we omit the percentage in the metric scores.

Compared methods. We first compare our method with two state-of-the-art (SOTA) image captioning methods, i.e., BUTD [2] and AoANet [21]. For the TextCap task, we compare our method with the current SOTA methods M4CCaptioner [40] and MMR-SA [45]. For fair comparisons, we use the same dataset annotation and multimodal feature extraction methods (including the OCR system and Faster $\mathrm{RCNN}$ ) for all considered methods in our experiments. We conduct extensive experiments on the validation and test set. In particular, the evaluation results are provided by the test server of the TextCaps-Challenge ${ }^{1}$. Since the number of submissions of the results on the test set is limited, we conduct ablation studies on the validation set.

\footnotetext{
${ }^{1}$ TextCaps: https://textvqa.org/textcaps
}

\begin{tabular}{llcccc}
\hline$\#$ & Method & Div-1 & Div-2 & selfCIDEr & CR \\
\hline 1 & BUTD & 27.0 & 36.4 & 45.3 & - \\
2 & M4C-Captioner & 27.2 & 41.2 & 49.4 & 27.3 \\
3 & Ours & $\mathbf{2 9 . 8}$ & $\mathbf{4 3 . 8}$ & $\mathbf{5 7 . 6}$ & $\mathbf{3 7 . 8}$ \\
\hline 4 & Human & 62.1 & 87.0 & 90.9 & 19.3 \\
\hline
\end{tabular}

Table 2. Diversity analysis. The BUTD and M4C-Captioner generate diverse captions via beam search (beam size is 5).

\begin{tabular}{clccccccc}
\hline$\#$ & Projection & B & M & R & S & C & A & F1 \\
\hline 1 & Single & 23.9 & 22.2 & 46.7 & 15.6 & 90.3 & 48.4 & 68.8 \\
2 & Multiple & 23.7 & 22.4 & 46.3 & 16.0 & 90.7 & 49.0 & 68.9 \\
3 & Sequence & $\mathbf{2 4 . 7}$ & $\mathbf{2 2 . 5}$ & $\mathbf{4 7 . 1}$ & $\mathbf{1 5 . 9}$ & $\mathbf{9 5 . 5}$ & $\mathbf{4 9 . 1}$ & $\mathbf{7 1 . 8}$ \\
\hline
\end{tabular}

Table 3. Ablation studies of anchor proposal module (AnPM) with independent projection (FC) and sequence projection (RNN). Apart from using captioning metrics, we also use accuracy (A) and F1 score to further measure the performance of AnPM.

\subsection{Implementation details}

In our implementation ${ }^{2}$, the feature dimension $d$ is 768 and the $f_{*}$ is a linear layer with LayerNorm activation function to stabilise training. We train our model for 12,000 iterations with a batch size of 128 . During training, we use the Adamax optimiser [23] with a learning rate of 2e-4. We adopt default parameter settings of BERT-BASE [12] for the transformer module $\Psi$, such as 12 self-attention heads. But the number of stacked layers are $L_{1}=2, L_{2}=L_{3}=4$, respectively. For fair comparisons, following the TextCaps benchmark [40], we use the same fixed common vocabulary and the feature embeddings of visual objects and OCR tokens. The number of visual objects is $N=100$ and the number of OCR tokens is $M=50$. The maximum generation length is $C=30$. The trade-off parameters of different losses are set to $\alpha=\beta=\gamma=1$.

\subsection{Main results}

Overall results. As shown in Table 1, we first compare our method with the current SOTA captioning models, including BUTD, AoANet, and M4C-Captioner. From the table, the BUTD and AoANet, standard image captioning models, show poor performances on the validation set since they fail to describe the texts in images. M4C-Captioner reasons over multimodal information and outperforms standard image captioning models by a large margin. Compared with M4C-Captioner, our model improves the CIDEr score from 89.6 to 95.5 on the validation set and achieves 6 absolute improvement on the test set. In particular, we also report the result of visual-captioner $\mathrm{AnCM}_{v}$ (row 5), which can be seen as a degraded version of our model without using OCR tokens. Same as M4C-Captioner w/o OCRs (row 4), $\mathrm{AnCM}_{v}$ is hard to generate reliable captions for the TextCaps dataset. To address this issue, our model is equipped with an additional text-captioner that refines gen-

\footnotetext{
${ }^{2}$ https://github.com/guanghuixu/AnchorCaptioner.
} 


\begin{tabular}{cccccccc}
\hline$\#$ & Anchor & ACG & B & M & R & S & C \\
\hline 1 & & All & 21.2 & 21.0 & 44.8 & 14.5 & 76.6 \\
2 & Large & Around & 21.4 & 21.1 & 44.9 & 14.4 & 77.4 \\
3 & & Random & 20.8 & 20.7 & 44.4 & 14.1 & 72.6 \\
\hline 4 & & All & 21.2 & 21.0 & 44.8 & 14.5 & 76.6 \\
5 & Centre & Around & 21.5 & 21.2 & 45.0 & 14.4 & 78.0 \\
6 & & Random & 20.7 & 20.8 & 44.5 & 14.1 & 73.1 \\
\hline 7 & & All & 21.1 & 21.1 & 44.7 & 14.6 & 76.7 \\
8 & - & Random & 20.4 & 20.6 & 44.1 & 13.9 & 70.2 \\
\hline 9 & & All & 23.5 & 22.4 & 46.3 & 15.7 & 90.3 \\
10 & GT & Around & 22.1 & 21.9 & 45.6 & 15.2 & 83.9 \\
11 & & Random & 21.4 & 21.2 & 45.0 & 14.7 & 78.7 \\
\hline 12 & AnPM & AnPM & 24.7 & 22.5 & 47.1 & 15.9 & 95.5 \\
13 & GT & GT & $\mathbf{2 5 . 6}$ & $\mathbf{2 3 . 4}$ & $\mathbf{4 8 . 1}$ & $\mathbf{1 6 . 9}$ & $\mathbf{1 0 4 . 9}$
\end{tabular}

Table 4. Ablation studies of ACG construction using rule-based approaches. For instance, row 2 ('Large'+'Around') means that we choose an OCR token with the largest region size as the anchor, and then group the five closest tokens to construct its ACG. In particular, we randomly group some tokens into an ACG, denoted as 'Random' in the table. 'GT' denotes ground-truth and 'AnPM' means using the prediction of AnPM.

erated captions with the text information. For fair comparisons, we choose the ACG with the highest anchor score to refine the generated caption in this experiment, since existing methods derive only one caption for each input image. In this way, our full model further boosts the CIDEr score from 29.1 to 95.5 in the validation set.

Diversity analysis. To further evaluate the diversity of the generated captions, we compare Anchor-Captioner with BUTD and M4C-Captioner in terms of diversity metrics. Since existing methods only generate a global description for an image, we use the beam search technique for them to produce diverse captions as baselines, where the beam size is set to 5. For fair comparisons, in our method, we also sample five ACGs for each image to generate captions. As shown in Table 2, our method surpasses baselines in terms of all considered metrics. Interestingly, the groundtruth captions (by humans) have high selfCIDEr but with low OCR cover ratio (CR). It means that humans may tend to describe the salient image contents but ignore some OCR tokens. Compared with human captioning, our method is able to generate multiple captions with content diversity, covering more OCR tokens. Note that, cover ratio (CR) score for BUTD method is empty, because OCR tools are not used in it.

\subsection{Ablation studies}

In this section, we further conduct ablation studies to demonstrate the effectiveness of AnPM and AnCM.

For AnPM, we first compare three different kinds of ACG construction strategies, i.e., independent projection (FC), multiple projection (transformer module) and sequence projection (RNN module). As shown in Table 3, the RNN outperforms FC in terms of all considered metrics,

\begin{tabular}{clccccc}
\hline$\#$ & Method & $\mathrm{B}$ & $\mathrm{M}$ & $\mathrm{R}$ & $\mathrm{S}$ & $\mathrm{C}$ \\
\hline 1 & M4C-Captioner & 23.3 & 22.0 & 46.2 & 15.6 & 89.6 \\
2 & M4C-Captioner $^{\dagger}$ & 24.1 & 22.6 & 46.7 & 15.7 & 93.8 \\
3 & M4C-Captioner* $^{*}$ & 24.4 & 22.6 & 46.9 & 15.8 & 99.6 \\
\hline 4 & AnCM $_{v}+$ AnCM $_{t}^{\dagger}$ & 24.7 & 22.5 & 47.1 & 15.9 & 95.5 \\
5 & AnCM $_{v}+$ AnCM $_{t}^{*}$ & $\mathbf{2 5 . 6}$ & $\mathbf{2 3 . 4}$ & $\mathbf{4 8 . 1}$ & $\mathbf{1 6 . 9}$ & $\mathbf{1 0 4 . 9}$ \\
\hline
\end{tabular}

Table 5. Ablation studies of Anchor Caption Module (AnCM). $\dagger$ denotes captioning modules using prediction ACGs provided by AnPM, while $*$ denotes captioning modules using ground-truth.

especially improves the CIDEr score from 90.3 to 95.5 . As discussed in Sec. 3.2, the sequence projection is more reasonable since it considers the history prediction. More details can be found in the supplement material. Moreover, we also report the accuracy of anchor prediction and the F1 score of the predicted ACG. Note that, there is a trade-off between obtaining high F1 score and diversity. To achieve high accuracy and F1 score, AnPM tends to predict the most frequently described ACG, which, however, could suffer from low diversity of generated captions.

In addition to the above comparisons, we also compare AnPM (RNN projection) with the rule-based ACG construction and report the quantitative results in Table 4. To be specific, we first adopt different rules to select token as an anchor, including the largest token (rows 1-3), the centre token (rows 4-6), the ground-truth anchor (rows 9-11). Then, we choose tokens to construct ACG using different strategies (i.e., 'All / Around / Random'). In particular, we try to group tokens into a graph directly without performing anchor selection process (in rows 7-8). From the table, all the rule-based methods suffer low metric performance even given the GT anchor to construct ACGs. The learning-based method (AnPM) outperforms rule-based methods by a large margin. One reason is that our AnPM considers the rich semantic information of the tokens themselves and the visual information in images, while the rule-based approaches mainly use shallow information such as size and location.

We also conduct ablation experiments for AnCM. From the results in Table 5, we draw the following main observations. 1) As shown in rows 1-3, the M4C-Captioner ${ }^{\dagger}$ and M4C-Captioner* that take the predicted ACGs and ground-truth ACGs as inputs, outperform the original M4CCaptioner by around 4 and 10 in terms of the CIDEr score, respectively. These results well verify our idea, i.e., first group OCR tokens into different ACG and then describe each ACG with a specific caption. 2) Compared with M4CCaptioner (row 1), our method improves CIDEr score from 89.6 to 95.5. 3) Equipped with AnPM, the M4C-Captioner ${ }^{\dagger}$ (row 2) achieves better performance, which implies that our AnPM can be easily extended to existing text-based reasoning methods. 4) Even for the same ACG inputs, our method is still superior to M4C-Captioner ${ }^{\dagger}$ and M4C-Captioner*, which demonstrates the powerful captioning ability of our 


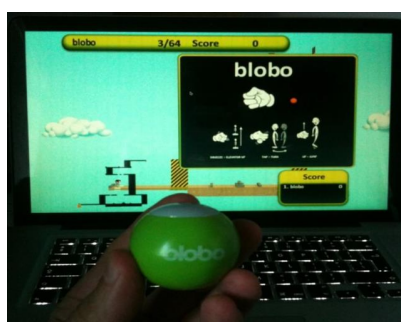

M4C: a person is holding a green laptop with a green screen that says blobo

$\overline{\mathbf{A n C M}}_{\mathbf{v}}$ : a person is holding a compu-ter computer monitor

Ours-1: a person is holding a blobo game on a computer screen

Ours-2: a blobo game is being held

in front of a computer screen

Ours-3: a computer screen shows a game called blobo

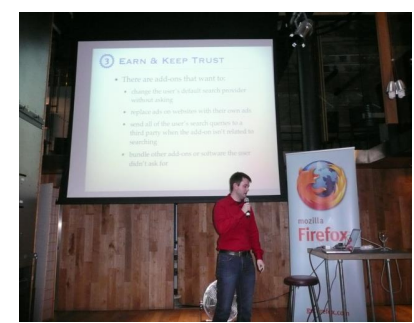

M4C: a man stands at a podium at a podium that says firefox

$\mathbf{A n C M}_{\mathbf{v}}$ : a man is standing a presentation on a screen that says

Ours-1: a man is giving a presentation with a screen that says mozilla

Ours-2: a man is giving a presentation with a screen that says " earn \& keep" Ours-3: a man is giving a presentation with a screen that says " earn your keep trust "

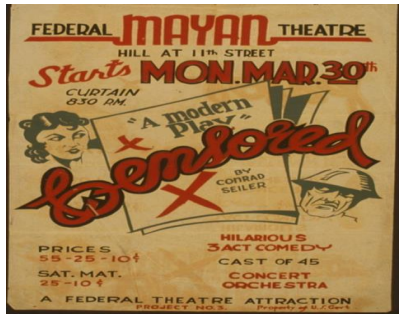

M4C: a poster for the mayan starts and theatre

AnCM $_{\mathbf{v}}$ : a poster for a $<$ unk $><$ unk $>$ shows a man of a man in the top

Ours-1: a poster for mayan theatre at the top of the page

Ours-2: a poster for mayan hill and 11 th street

Ours-3: a poster for mayan and theatre shows a picture of a man on the bottom

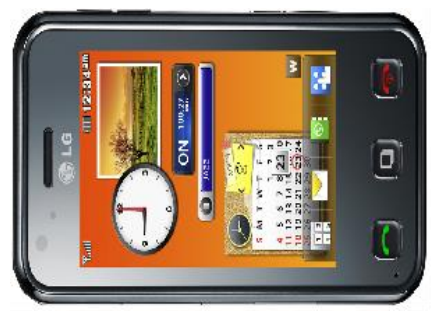

M4C: a phone with the word $1 \mathrm{~g}$ on the screen

$\mathbf{A n C M}_{\mathbf{v}}$ : a orange phone with a red of a phone and a sign that says it

Ours-1: an orange $\lg$ phone with a screen that says ' $\underline{\lg }$ ' on it

Ours-2: an orange phone with the word jazz on it

Ours-3: a phone screen shows a time of $567: 00$ on it

Figure 3. Visualisation results on the TextCaps validation set. The prediction results of M4C-Captioner (M4C), visual-captioner (AnCM M $_{v}$ and the proposed Anchor-Captioner are placed below the images in turn. The $<$ unk $>$ denotes 'unkown' token. For better visualisation,

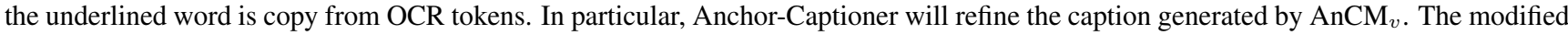
tokens are viewed in red colour.

AnCM. 5) According to the last two rows, our AnCM suffers a performance degradation with the predicted ACGs as input, indicating that our method still has great potentials for improving.

\subsection{Visualisation analysis}

To further demonstrate the effectiveness of our method, we show some visualisation results on the TextCaps validation set. From Figure 3, our Anchor-Captioner is able to refine the rough captions generated by the visual-captioner $\left(\mathrm{AnCM}_{v}\right)$. Specifically, for each input image, $\mathrm{AnCM}_{v}$ first uses visual information to generate a global caption, such as 'a man' and 'a poster'. Similar to general image captioning models, $\mathrm{AnCM}_{v}$ is difficult to describe the texts in images. As a result, we can see the visual-specific captions may contain some $<$ unk $>$ tokens. It means that $\mathrm{AnCM}_{v}$ cannot use limited information to generate reasonable predictions in this case. And then, Anchor-Captioner use anchorcentred graphs (ACGs) to further refine the visual-specific captions. Note that, the refine process is not only to replace the $<$ unk $>$ token, but also to revise the entire caption. There are $66.39 \%$ of generated captions with $<$ unk $>$, and each caption has $1.24<$ unk $>$ on average. AnCM $_{t}$ modified $26.85 \%$ of words on the $\mathrm{AnCM}_{v}$ 's output and improved CIDEr score from 29.1 to 95.5 (see Tabel 1). We also randomly sample different ACGs to demonstrate the diversity of our generation. Compared with M4C-Captioner, our method is able to generate fine-grained captions and cover more OCR tokens. To further demonstrate the controllability and diversity of our method, we provide more visualisation results in the supplement material.

\section{Conclusion}

In this paper, we have proposed an Anchor-Captioner to resolve the TextCap task. To solve this task, existing methods tend to generate only one rough global caption which contains one or two salient objects in the complex scene. Intuitively, such methods may ignore some regions that people are really interested in. Unlike existing methods, we seek to generate multiple captions from different views and cover more valuable scene information. Specifically, we first propose an anchor proposal module to group OCR tokens and construct anchor-centred graphs (ACGs) by modelling the relationship between image contents. After that, our anchor captioning module first generates a rough visualspecific caption and then uses the above ACGs to further refine it to multiple text-specific captions. In this way, our method is able to generate diverse captions to cover more information in images. Our method achieves state-of-theart performance on the TextCaps dataset and outperforms the benchmark by 6 in terms of CIDEr score. Extensive ablation experiments also verify the effectiveness of each component of our method. Note that our anchor captioning module has the potential to solve both image captioning and text-based image captioning tasks simultaneously, which we leave to our future study.

Acknowledgements. This work was partially supported by the Science and Technology Program of Guangzhou, China, under Grant 202007030007, Fundamental Research Funds for the Central Universities D2191240, Program for Guangdong Introducing Innovative and Enterpreneurial Teams 2017ZT07X183, Opening Project of Guangdong Key Laboratory of Big Data Analysis and Processing. 


\section{References}

[1] Peter Anderson, Basura Fernando, Mark Johnson, and Stephen Gould. Spice: Semantic propositional image caption evaluation. In ECCV, pages 382-398, 2016.

[2] Peter Anderson, X. He, C. Buehler, Damien Teney, Mark Johnson, Stephen Gould, and Lei Zhang. Bottom-up and top-down attention for image captioning and visual question answering. In CVPR, pages 6077-6086, 2018.

[3] Lei Jimmy Ba, Jamie Ryan Kiros, and Geoffrey E. Hinton. Layer normalization. In CoRR, 2016.

[4] Youngmin Baek, Bado Lee, Dongyoon Han, Sangdoo Yun, and Hwalsuk Lee. Character region awareness for text detection. In CVPR, pages 9365-9374, 2019.

[5] Ali Furkan Biten, Ruben Tito, Andrés Mafla, Lluís Gómez, M. Rusiñol, Ernest Valveny, C. Jawahar, and Dimosthenis Karatzas. Scene text visual question answering. In $I C C V$, pages 4290-4300, 2019.

[6] Fedor Borisyuk, Albert Gordo, and Viswanath Sivakumar. Rosetta: Large scale system for text detection and recognition in images. In ACM SIGKDD, pages 71-79. ACM, 2018.

[7] Shizhe Chen, Qin Jin, Peng Wang, and Qi Wu. Say as you wish: Fine-grained control of image caption generation with abstract scene graphs. In CVPR, pages 9959-9968, 2020.

[8] Marcella Cornia, Lorenzo Baraldi, and Rita Cucchiara. Show, control and tell: A framework for generating controllable and grounded captions. In $C V P R$, pages 8307-8316, 2019.

[9] Chaorui Deng, Ning Ding, Mingkui Tan, and Qi Wu. Length-controllable image captioning. In $E C C V$, volume abs/2007.09580, 2020.

[10] Michael J. Denkowski and A. Lavie. Meteor universal: Language specific translation evaluation for any target language. In WMT-ACL, pages 376-380, 2014.

[11] Aditya Deshpande, Jyoti Aneja, Liwei Wang, Alexander G. Schwing, and David A. Forsyth. Fast, diverse and accurate image captioning guided by part-of-speech. In $C V P R$, pages 10695-10704, 2019.

[12] Jacob Devlin, Ming-Wei Chang, Kenton Lee, and Kristina Toutanova. Bert: Pre-training of deep bidirectional transformers for language understanding. In NAACL-HLT, pages 4171-4186, 2019.

[13] I. Fine. Sensory systems: Do you hear what i see? Nature, 508:461-462, 2014.

[14] Chuang Gan, Zhe Gan, X. He, Jianfeng Gao, and L. Deng. Stylenet: Generating attractive visual captions with styles. In CVPR, pages 955-964, 2017.

[15] Zhe Gan, Chuang Gan, X. He, Y. Pu, K. Tran, Jianfeng Gao, L. Carin, and L. Deng. Semantic compositional networks for visual captioning. In CVPR, pages 1141-1150, 2017.

[16] Marjan Ghazvininejad, Omer Levy, Yinhan Liu, and Luke Zettlemoyer. Mask-predict: Parallel decoding of conditional masked language models. In EMNLP-IJCNLP, pages 61116120, 2019.

[17] Longteng Guo, J. Liu, Peng Yao, Jiangwei Li, and H. Lu. Mscap: Multi-style image captioning with unpaired stylized text. In CVPR, pages 4204-4213, 2019.
[18] Yong Guo, Yin Zheng, Mingkui Tan, Qi Chen, Jian Chen, Peilin Zhao, and Junzhou Huang. Nat: Neural architecture transformer for accurate and compact architectures. In Advances in Neural Information Processing Systems, pages 735-747, 2019.

[19] Ronghang Hu, Amanpreet Singh, Trevor Darrell, and Marcus Rohrbach. Iterative answer prediction with pointeraugmented multimodal transformers for textvqa. In $C V P R$, pages 9992-10002, 2020.

[20] Deng Huang, Peihao Chen, Runhao Zeng, Qing Du, Mingkui Tan, and Chuang Gan. Location-aware graph convolutional networks for video question answering. In The AAAI Conference on Artificial Intelligence (AAAI), 2020.

[21] Lun Huang, Wenmin Wang, J. Chen, and Xiao-Yong Wei. Attention on attention for image captioning. In ICCV, pages 4633-4642, 2019.

[22] Justin Johnson, Andrej Karpathy, and Li Fei-Fei. Densecap: Fully convolutional localization networks for dense captioning. In $C V P R$, pages 4565-4574, 2016.

[23] Diederik P Kingma and Jimmy Ba. Adam: A method for stochastic optimization. In ICLR, pages 4190-4198, 2014.

[24] Ranjay Krishna, Yuke Zhu, Oliver Groth, Justin Johnson, Kenji Hata, Joshua Kravitz, Stephanie Chen, Yannis Kalantidis, Li-Jia Li, David A. Shamma, Michael S. Bernstein, and Li Fei-Fei. Visual genome: Connecting language and vision using crowdsourced dense image annotations. IJCV, 123:3273, 2016.

[25] Jason Lee, Elman Mansimov, and Kyunghyun Cho. Deterministic non-autoregressive neural sequence modeling by iterative refinement. In $E M N L P$, volume abs/1802.06901, pages 1173-1182, 2018.

[26] Jiwei Li, Michel Galley, Chris Brockett, Jianfeng Gao, and Bill Dolan. A diversity-promoting objective function for neural conversation models. In HLT-NAACL, pages 110-119, 2016.

[27] Chin-Yew Lin. Rouge: A package for automatic evaluation of summaries. In $A C L$, pages 74-81, 2004.

[28] Tsung-Yi Lin, Michael Maire, Serge J. Belongie, James Hays, Pietro Perona, Deva Ramanan, Piotr Dollár, and C. Lawrence Zitnick. Microsoft COCO: common objects in context. In ECCV, pages 740-755, 2014.

[29] L. Liu, Mengge He, G. Xu, Mingkui Tan, and Qi Wu. How to train your agent to read and write. ArXiv, abs/2101.00916, 2021.

[30] Siqi Liu, Zhenhai Zhu, Ning Ye, Sergio Guadarrama, and Kevin Murphy. Improved image captioning via policy gradient optimization of spider. In ICCV, pages 873-881, 2017.

[31] Yuliang Liu, Hao Chen, Chunhua Shen, Tong He, Lianwen Jin, and Liangwei Wang. Abcnet: Real-time scene text spotting with adaptive bezier-curve network. In $C V P R$, pages 9806-9815, 2020.

[32] Jiasen Lu, Jianwei Yang, Dhruv Batra, and D. Parikh. Neural baby talk. 2018 IEEE/CVF Conference on Computer Vision and Pattern Recognition, pages 7219-7228, 2018.

[33] Alexander Patrick Mathews, Lexing Xie, and Xuming He. Semstyle: Learning to generate stylised image captions using unaligned text. In CVPR, pages 8591-8600, 2018. 
[34] A. Mishra, Shashank Shekhar, A. Singh, and A. Chakraborty. Ocr-vqa: Visual question answering by reading text in images. ICDAR, pages 947-952, 2019.

[35] Shuaicheng Niu, J. Wu, Yi-Fan Zhang, Yong Guo, P. Zhao, Junzhou Huang, and Mingkui Tan. Disturbanceimmune weight sharing for neural architecture search. ArXiv, abs/2003.13089, 2020.

[36] Kishore Papineni, Salim Roukos, Todd Ward, and Wei-Jing Zhu. Bleu: A method for automatic evaluation of machine translation. In ACL, page 311-318, 2002.

[37] Colin Raffel, Noam Shazeer, Adam Roberts, Katherine Lee, Sharan Narang, Michael Matena, Yanqi Zhou, Wei Li, and Peter J. Liu. Exploring the limits of transfer learning with a unified text-to-text transformer. JMLR, 21(140):1-67, 2020.

[38] Shaoqing Ren, Kaiming He, Ross B. Girshick, and J. Sun. Faster r-cnn: Towards real-time object detection with region proposal networks. TPAMI, 39:1137-1149, 2017.

[39] Steven J. Rennie, Etienne Marcheret, Youssef Mroueh, Jerret Ross, and Vaibhava Goel. Self-critical sequence training for image captioning. In CVPR, pages 1179-1195, 2017.

[40] Oleksii Sidorov, Ronghang Hu, Marcus Rohrbach, and Amanpreet Singh. Textcaps: A dataset for image captioning with reading comprehension. In $E C C V$, pages 742-758, 2020.

[41] Amanpreet Singh, Vivek Natarjan, Meet Shah, Yu Jiang, Xinlei Chen, Devi Parikh, and Marcus Rohrbach. Towards vqa models that can read. In CVPR, pages 8317-8326, 2019.

[42] Ashish Vaswani, Noam Shazeer, Niki Parmar, Jakob Uszkoreit, Llion Jones, Aidan N. Gomez, Lukasz Kaiser, and Illia Polosukhin. Attention is all you need. In NeurIPS, pages 5998-6008, 2017.

[43] Ramakrishna Vedantam, C. Lawrence Zitnick, and Devi Parikh. Cider: Consensus-based image description evaluation. In CVPR, pages 4566-4575, 2015.

[44] Oriol Vinyals, Alexander Toshev, Samy Bengio, and Dumitru Erhan. Show and tell: A neural image caption generator. In CVPR, pages 3156-3164, 2015.

[45] Jing Wang, Jinhui Tang, and Jiebo Luo. Multimodal attention with image text spatial relationship for ocr-based image captioning. In ACM MM, page 4337-4345, 2020.

[46] Qingzhong Wang and Antoni B. Chan. Describing like humans: On diversity in image captioning. In $C V P R$, pages 4195-4203, 2019.

[47] Ronald J Williams. Simple statistical gradient-following algorithms for connectionist reinforcement learning. Machine learning, 8(3-4):229-256, 1992.

[48] Yingce Xia, Fei Tian, Lijun Wu, Jianxin Lin, Tao Qin, Nenghai Yu, and Tie-Yan Liu. Deliberation networks: Sequence generation beyond one-pass decoding. In NeurIPS, pages 1784-1794, 2017.

[49] Kelvin Xu, Jimmy Ba, Ryan Kiros, Kyunghyun Cho, Aaron C. Courville, R. Salakhutdinov, R. Zemel, and Yoshua Bengio. Show, attend and tell: Neural image caption generation with visual attention. In $I C M L$, volume abs/1502.03044, pages 2048-2057, 2015.

[50] Runhao Zeng, Haoming Xu, Wenbing Huang, Peihao Chen, Mingkui Tan, and Chuang Gan. Dense regression network for video grounding. In The IEEE Conference on Computer Vision and Pattern Recognition (CVPR), June 2020.

Supplementary Materials: Towards Accurate Text-based Image Captioning with Content Diversity Exploration

Guanghui $\mathrm{Xu}^{1,2}$ Authors contributed equally., Shuaicheng $\mathrm{Niu}^{1 *}$, Mingkui $\mathrm{Tan}^{1,4}$, Yucheng $\mathrm{Luo}^{1}$, Qing $\mathrm{Du}^{1,4}$ Corresponding author, Qi $\mathrm{Wu}^{3}$

${ }^{1}$ South China University of Technology, ${ }^{2}$ Pazhou Laboratory, ${ }^{3}$ University of Adelaide

${ }^{4}$ Key Laboratory of Big Data and Intelligent Robot, Ministry of Education,

sexuguanghui@mail.scut.edu.cn, \{mingkuitan, duqing\}@scut.edu.cn, qi.wu01@adelaide.edu. au 


\section{Supplementary Materials}

In the supplementary, we provide more implementation details and experimental results of the proposed Anchor-Captioner. We organise the supplementary as follows.

- In Section A, we provide the detailed training and inference algorithms of Anchor-Captioner.

- In Section B, we give more discussions on the anchor-centred graph (ACG) construction strategy.

- In Section C, we conduct more ablation experiments to verify the generalisation ability of Anchor-Captioner.

- In Section D, we conduct more ablation experiments to further measure the importance of each loss term.

- In Section E, we show more visualisation results to further verify the promise of the proposed method.

\section{A. Algorithms}
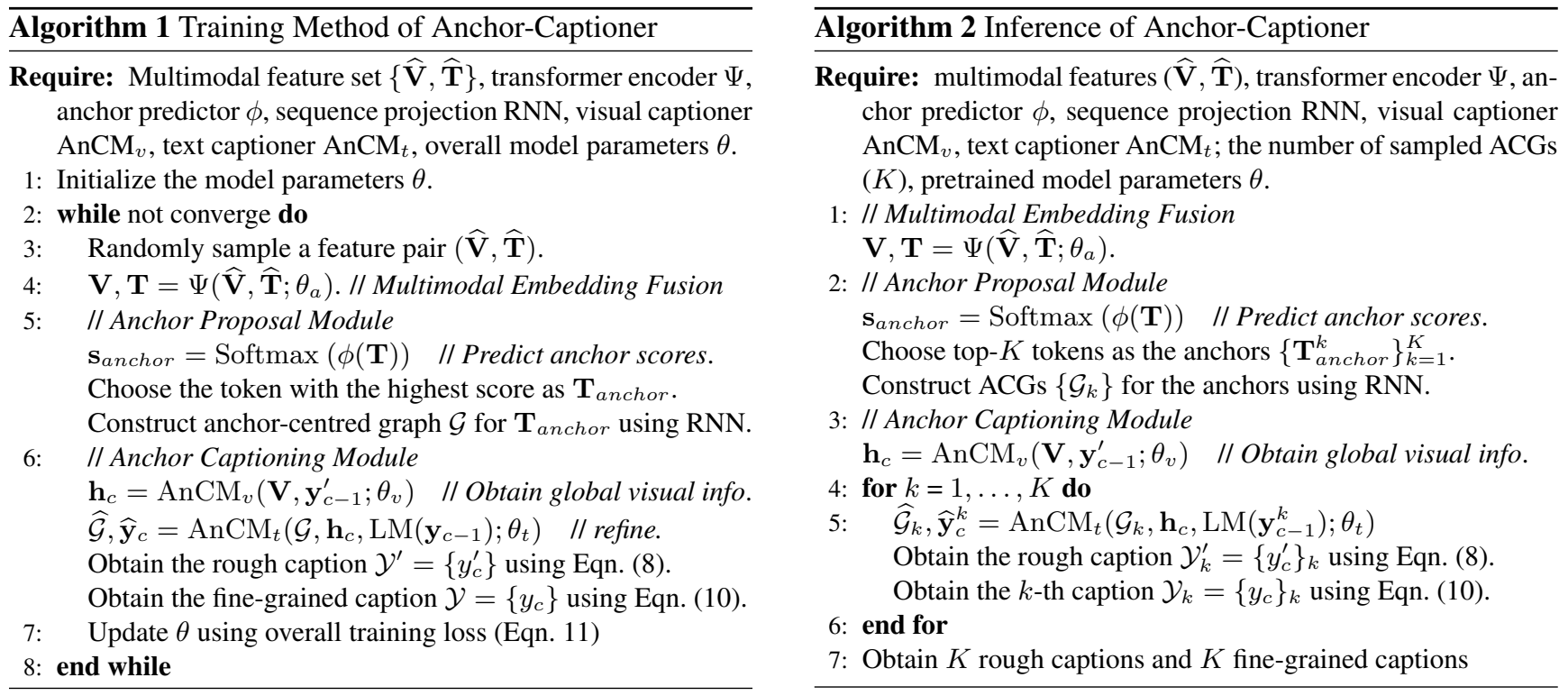

In this section, we provide the detailed training and inference algorithms of our method in Algorithms 1 and 2. Given an input image, we first fuse visual and text features to obtain multimodal embeddings. Then, we apply the anchor proposal module (AnPM) to choose and group texts to construct a series of anchor-centred graphs (ACGs), where each ACG denotes a group of relevant OCR tokens that are used to generate a specific caption. Last, we employ the visual- captioner ( $\left.\mathrm{AnCM}_{v}\right)$ to generate a rough caption and then use ACGs as guidance to refine the generated caption by the text-captioner $\left(\mathrm{AnCM}_{t}\right)$. In particular, we adopt the top-1 ACG for the training while using top-K ACGs to generate K diverse captions in the inference.

\section{B. More details about ACG}

As shown in Figure S1, to construct an anchor-centred graph (ACG), we mainly consider three kinds of construction strategies, i.e., independent projection (fully connected layer), multiple projection (4-layer Transformer module) and sequence projection (RNN module). Specifically, based on a given anchor $\mathbf{T}_{\text {anchor }}$, the independent projection directly predicts a correlation score for each token without considering others, while the multiple projection considers global information via self-attention mechanism. As shown in Figure S1, the multiple projection makes prediction for a token (e.g., $\mathbf{T}_{1}$ ) using rich neighbourhood information. In particular, the sequence projection considers the relationships among $\mathbf{T}_{\text {anchor }}$, all OCR tokens and history predictions. The order of OCR tokens is determined by the ranking of confidence scores (in descending order) obtained from the OCR model. In a word, the three strategies use different information to construct ACGs, where the independent and multiple projection mainly consider local and global information, and the sequence projection perceives previous predictions through hidden state. To some extent, the sequence projection will be more reasonable, because the choice between different tokens to construct an ACG is not completely independent. To train AnPM, we first parse ground-truth (gt) captions into candidate sets, where gt-anchor is the most frequently described token and gt-ACG are the tokens appeared in 


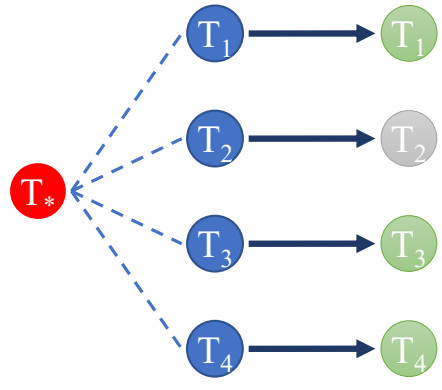

(a) independent projection

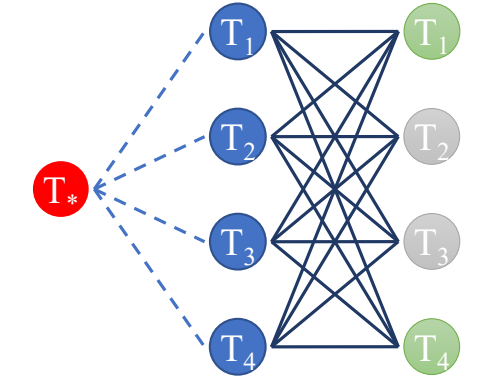

(b) multiple projection

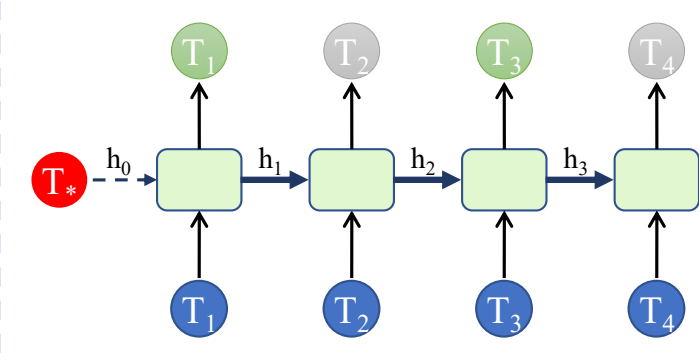

(c) sequence projection

Figure S1. Illustrations of different ACG construction strategies. The red circle denotes the anchor, and the blue circles denote the candidate OCR tokens to be selected. After performing prediction, the green one indicates that the current token and the anchor belong to the same group of ACG, while the gray is the opposite. It means that the ACGs in (a-c) are $\left\{\mathrm{T}_{*}, \mathrm{~T}_{1}, \mathrm{~T}_{3}, \mathrm{~T}_{4}\right\},\left\{\mathrm{T}_{*}, \mathrm{~T}_{1}, \mathrm{~T}_{4}\right\}$ and $\left\{\mathrm{T}_{*}, \mathrm{~T}_{1}, \mathrm{~T}_{3}\right\}$, respectively.

\begin{tabular}{|c|c|c|c|c|c|c|c|c|}
\hline \# & Method & trained on & evaluated on & BLEU & METEOR & ROUGE_L & SPICE & $\overline{\text { CIDEr }}$ \\
\hline 1 & \multirow{6}{*}{ M4C } & \multirow{2}{*}{$\mathrm{COCO}$} & $\mathrm{COCO}$ & 34.3 & 27.5 & 56.2 & 20.6 & 112.2 \\
\hline 2 & & & TextCaps & 12.3 & 14.2 & 34.8 & 9.2 & 30.3 \\
\hline 3 & & \multirow{2}{*}{ TextCa } & $\mathrm{COCO}$ & 8.3 & 15.1 & 34.2 & 8.0 & 17.3 \\
\hline 4 & & & TextCaps & 23.3 & 22.0 & 46.2 & 15.6 & 89.6 \\
\hline 5 & & \multirow{2}{*}{ COCO+TextCaps } & $\mathrm{COCO}$ & 27.1 & 24.1 & 51.6 & 17.4 & 87.5 \\
\hline 6 & & & TextCaps & 21.9 & 22.0 & 45.0 & 15.6 & 84.6 \\
\hline 7 & \multirow{6}{*}{ Ours } & \multirow{2}{*}{$\mathrm{COCO}$} & $\mathrm{COCO}$ & 34.6 & 27.3 & 56.1 & 20.2 & 110.3 \\
\hline 8 & & & TextCaps & 12.6 & 13.8 & 35.2 & 8.8 & 29.2 \\
\hline 9 & & \multirow{2}{*}{ TextCaps } & $\mathrm{COCO}$ & 8.9 & 15.5 & 34.7 & 8.3 & 18.4 \\
\hline 10 & & & TextCaps & 24.7 & 22.5 & 47.1 & 15.9 & 95.5 \\
\hline 11 & & \multirow{2}{*}{ COCO+TextCaps } & $\mathrm{COCO}$ & 30.5 & 25.2 & 53.6 & 18.4 & 96.3 \\
\hline 12 & & & TextCaps & 23.6 & 22.2 & 46.2 & 15.7 & 90.0 \\
\hline
\end{tabular}

Table S1. More experiments about generalisation. We train our model and M4C-Captioner on TextCaps and COCO captioning training split and then evaluate the models on the different validation split. Specifically, 'COCO+TextCaps' denotes that a model uses both COCO captioning and TextCaps dataset for joint training. In practice, since the scale of COCO is much larger than TextCaps, we set the sampling rate to 1:8 to sample TextCaps as frequently as COCO.

the same caption. Here, we do not have semantic labels of the visual objects in an image, and thus we have trouble to know what objects are included in the ground-truth captions. In this work, we only consider the OCR token and its relative tokens to construct ACGs. We will consider training AnPM to propose sub-regions as RPN in the future study.

\section{More ablation studies about generalisation}

To further demonstrate the generalisation ability of our method, we conduct ablation experiments on COCO captioning and TextCaps dataset. COCO captioning is a famous large-scale dataset for general image captioning and TextCaps dataset is recently proposed to enhance the captioning ability of existing methods, especially the reading ability. As discussed in the main paper, general image captioning methods mainly focus on visual objects and overall scenes in images, while ignoring text information that is of critical importance for comprehensively understanding images. In this sense, it is necessary to study generalisation ability of existing methods on COCO captioning and TextCaps dataset. To this end, we exploit different settings to conduct experiments. From the results in Table S1, we draw the following main observations: 1) When only training models on COCO captioning (rows 1-2 and 7-8), our model achieves comparable performance as M4C-Captioner. 2) When training models on TextCaps dataset (rows 3-4 and 9-10), our model outperforms M4C-Captioner in terms of two evaluation settings. 3) When jointly training models using both COCO and TextCaps dataset (rows 5-6 and 11-12), our model improves the CIDEr score from $87.5 \%$ to $96.3 \%$ on COCO and achieves 5\% absolute improvement on TextCaps. 4) Unfortunately, as shown in rows 5-6 and 11-12, training on 'COCO+TextCaps' leads to worse performance than only using $\mathrm{COCO} /$ TextCaps (rows $1,4,7,10$ ). It means that simply improving the sampling ratio of these two datasets can not handle 

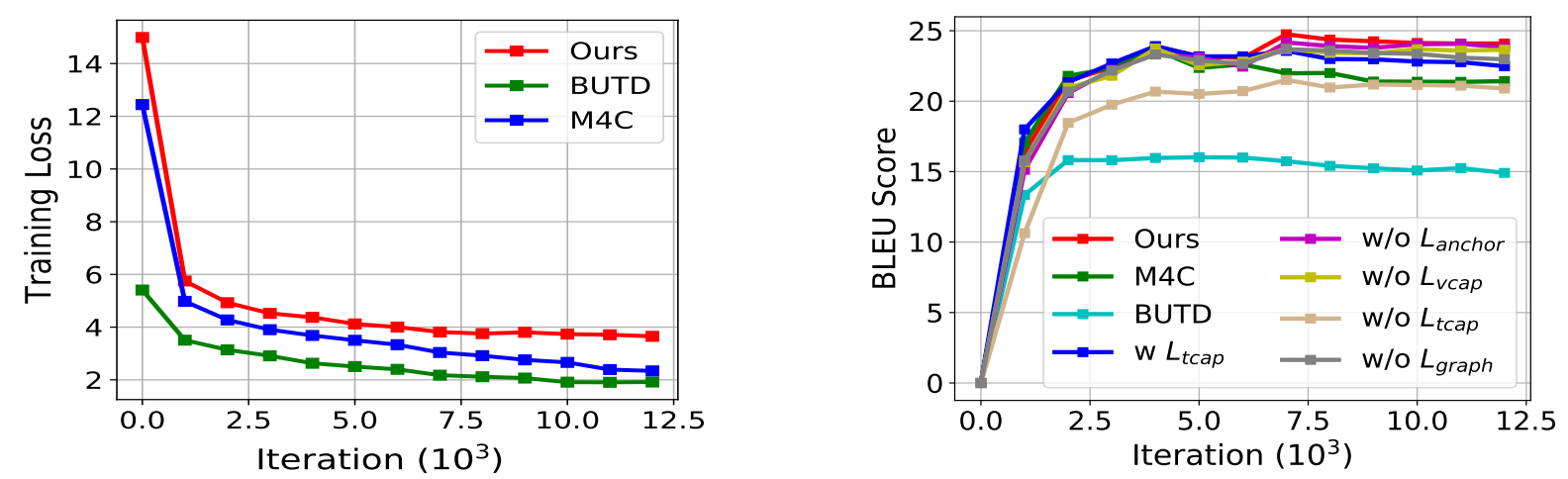

Figure S2. Left: The training loss of different methods on the TextCaps training set. Right: The BLEU scores under different iterations on the TextCaps validation set. The 'M4C' denotes M4C-Captioner model. The 'w/o' denotes 'Ours' without a specific loss term. For instance, 'w/o $\mathcal{L}_{\text {tcap }}$ ' denotes our method without $\mathcal{L}_{\text {tcap }}$ while 'w $\mathcal{L}_{\text {tcap }}$ ' denotes our method only using $\mathcal{L}_{\text {tcap }}$.

the domain shift problem, which is already a quite challenging task. However, combining COCO and TextCaps datasets for training is more suitable for complex real scenarios. In this way, the well-trained model is able to 'watch' visual objects and 'read' texts in images.

\section{More ablation studies about losses}

As shown in Figure S2, we also compare our method with baselines in terms of the training loss and the BLEU score. Since our total training loss contains four terms $\mathcal{L}_{\{\text {anchor, graph, vcap }, t c p\}}$, the value of our training loss is little higher than the compared methods. Our method tends to converge after $7 \mathrm{k}$ iteration and achieves the highest BLEU score on the validation set. Compared with the considered methods, our method has better generalisation ability to overcome the overfitting problem. To further measure the importance of losses in our method, we conduct several ablation studies, such as removing each loss term. In particular, we can train our model in an end-to-end miner (only using $\mathcal{L}_{\text {tcap }}$ ). From the results, the importance of the losses can be formulated as: $\mathcal{L}_{\text {tcap }}>\mathcal{L}_{\text {vcap }}>\mathcal{L}_{\text {graph }}>\mathcal{L}_{\text {anchor }}$.

\section{E. More visualisation analysis}

In this section, to further measure the qualities of our method's generation, we provide more visualisation results on TextCaps validation set. Specifically, we fist show more successful results of our method in Figure S3. Then, demonstrate the controllability of our method in Figure S4. Last but not least, in Figure S5, we provide some typical failure cases to evaluate our method more objectively.

As shown in Figure S3, compared with M4C-Captioner, our method is able to describe images from different views and cover more OCR tokens, represented as 'Ours-*'. In particular, our proposed AnCM is a progressive captioning module that $\mathrm{AnCM}_{v}$ first adopts visual information to generate a global caption and then $\mathrm{AnCM}_{t}$ refines the caption based on the text information of ACGs. Note that, the refining process not only to simply replace <unk $>$ token but also to revise the entire caption in terms of language syntax and contents. In addition, extensive experiments in the main paper also demonstrate the effectiveness of our method.

Based on anchor-centred graphs (ACGs), our method is able to generate multiple controllable image captions. To demonstrate the controllability of our method, we provide more visualisation about the generated captions aligned with the ACGs . As shown in Figure S4, the generated caption of $\mathrm{AnCM}_{t}$ is aligned with the ACGs. We also can see that the generated captions always contain anchors. One possible reason is that our model takes the most important OCR token as an anchor while the other tokens in ACG are used to aggregate information to the anchor. And thus the generated caption is supposed to be at least anchor-related.

As shown in Figure S5, we also provide typical failure cases to further analyse the performance of our method. 1) Although some images could be correctly describe via one global caption, our model still tends to output multiple diverse captions, which might be correct but uninformative, such as 'ruler ... has number 10/20' in (a). 2) Due to dataset bias, the model tends to generate words with high frequency in training set, such as brand name is iphone or lg in (b). 3) More critical, our model is sensitive to anchor-centred graphs (ACGs). As shown in (c)-(d), if the OCR recognise system fails to detect or only detects few OCR tokens in an image, our model will be degraded to existing models that only generate a global caption since we have trouble contracting different ACGs. 


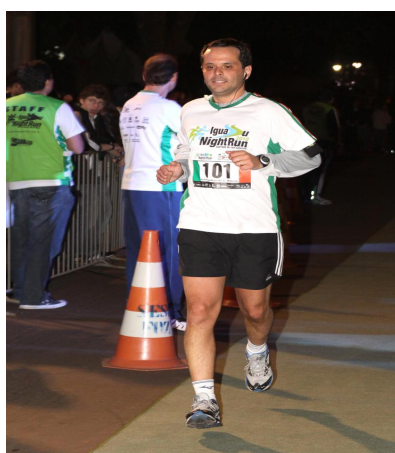

M4C: a man wearing a jersey that says igua nightrun on it

AnCM $\mathbf{M}_{\mathbf{v}}$ : a man in a white shirt green shirt with the number $<$ unk $>$ on it

Ours-1: a man in a white and green shirt with the number 101 on it Ours-2: a man in a white and green shirt with the word igua on it

Ours-3: a man in a white and green shirt with the number 8 on it

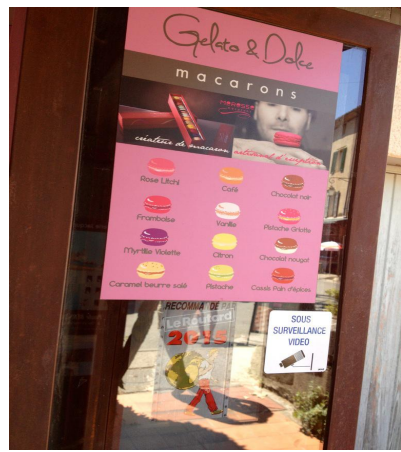

M4C: a display of pink and pink pink pink pink pink pink pink sign with the word macarons on it

$\operatorname{AnCM}_{\mathbf{v}}$ : a pink sign that $<$ unk $>$ pink pink called $<$ unk $>\&<$ unk $>$

Ours-1: a pink sign for a pink product called caramel de surveillance

Ours-2: a pink sign with a pink background that says ' myrtlle griotte ' on it

Ours-3: a pink sign with a pink background that says ' sous ' on it

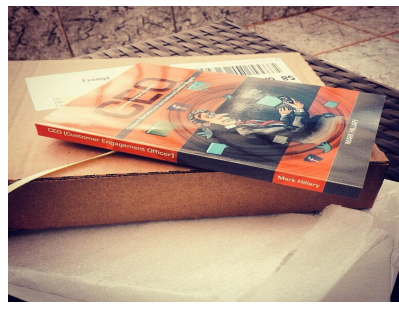

M4C: a stack of books with one titled ' exempt '

AnCM $_{\mathbf{v}}$ : a book titled $<$ unk $><$ unk $>$ sits on a table

Ours-1: a book by mark hillery is on a table

Ours-2: a box that says ' ceo ' on it Ours-3: a book titled " the author of the author of the book"

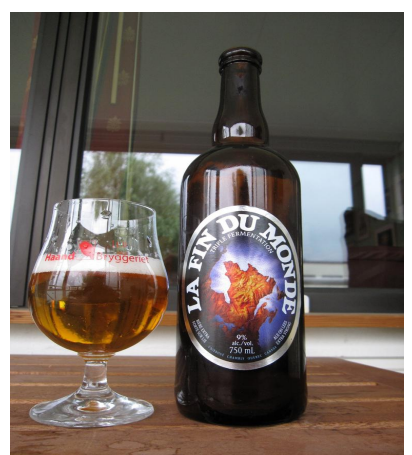

M4C: a bottle of $\underline{\mathrm{du}} \underline{\mathrm{du}} \underline{\mathrm{du}} \underline{\mathrm{du}} \underline{\mathrm{du}}$ $\underline{\mathrm{du}} \underline{\mathrm{du}}$

$\mathbf{A n C M}_{\mathbf{v}}$ : a bottle of $<$ unk $>$ beer $<$ unk $>$ sits next to a glass

Ours-1: a bottle of bryggeriet du ferme sits next to a glass of beer

Ours-2: a bottle of du ferme sits next to a glass of beer

Ours-3: a bottle of beer with the word " mat " on the label

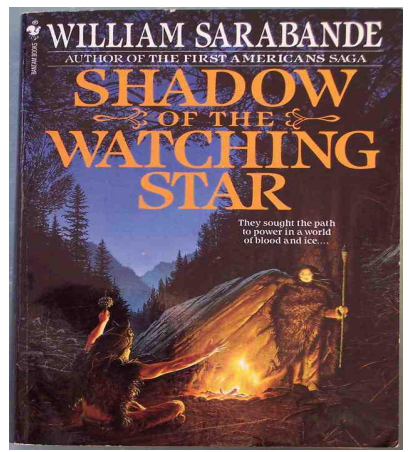

M4C: a book cover for the shadow watching watching by william sarabande

$\mathbf{A n C M}_{\mathbf{v}}$ : a book by for the book by the book by

Ours-1: a book cover for the shadow of the watching star

Ours-2: a book by william sarabande titled shadow of the first saga

Ours-3: a book titled " the best of the best of america "

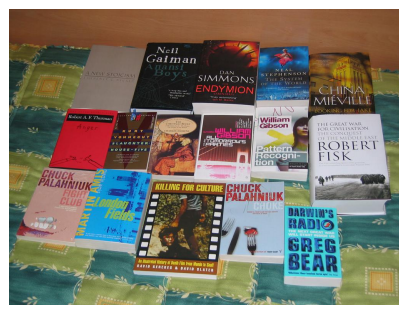

M4C: a table with books including one by chuck gaiman

AnCM $\mathbf{M}_{\mathbf{v}}$ : a collection of books including one titled them titled ' the

Ours-1: a collection of books with one of them titled ' darwin '

Ours-2: a collection of books with one of them titled ' neil gaiman ' Ours-3: a collection of books with one titled ' club ' by the author of the books

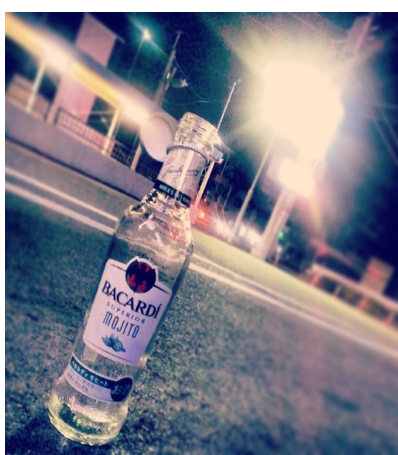

M4C: a bottle of racardi mojito mojito is on a table

$\operatorname{AnCM}_{\mathbf{v}}$ : a bottle of $<$ unk $>$ is $<$ unk $>$ sits on a table day

Ours-1: a bottle of bacardi superior mojito is on a sunny day

Ours-2: a bottle of bacardi is sitting on a pole

Ours-3: a bottle of alcohol with a label that says ' superior ' on it

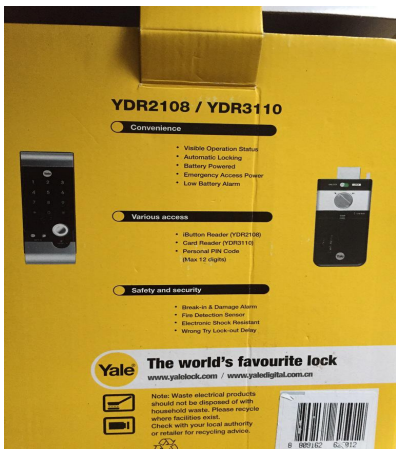

M4C: a yellow and yellow advertisement for a phone that says " the world 's "

$\operatorname{AnCM}_{\mathbf{v}}$ : a yellow that the words " $<$ unk $>$ 's $<$ unk $><$ unk $>$ on it

Ours-1: a yellow poster with the words the world 's favourite lock on it

Ours-2: a yellow and yellow sign that says ydr2108 / ydr3110 on it

Ours-3: a yellow and yellow poster for a yale should

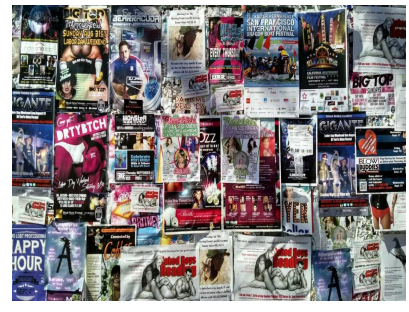

M4C: a display of magazines including one for the trammyshack

AnC $\mathbf{M}_{\mathbf{v}}$ : a display of posters including one that says ' $<$ unk $>$ ' on it Ours-1: a wall of posters with one that says ' big.top ' on it

Ours-2: a display of different posters including one that says ' trammyshack ' on it

Ours-3: a display of different types 14 of different types of which are labeled as ' connectedby'

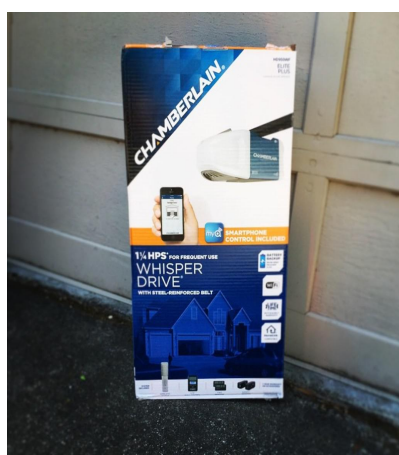

M4C: a phone that has the word whisper on it

$\overline{\mathbf{A n C M}}_{\mathbf{v}}$ : a box for a phone called the <unk>

Ours-1: a box for a phone called whisper drive

Ours-2: a box for a nokia phone has a phone number on it

Ours-3: a box for a phone called the phone

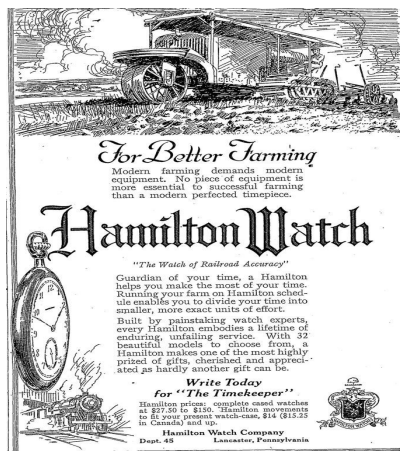

M4C: a poster for a watch that says ' better farming ' on it

$\mathbf{A n C M}_{\mathbf{v}}$ : a old advertisement and white advertisement for a watch called $<$ unk $><$ unk $>$

Ours-1: an old black and white advertisement for a watch for better farming

Ours-2: an old advertisement for a watch for better farming

Ours-3: a black and white page with the word " built " on it

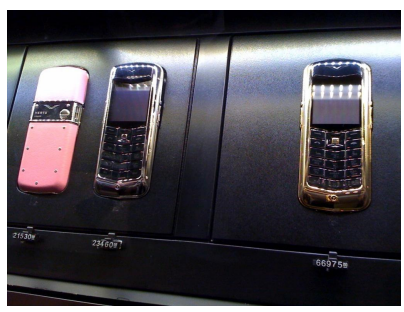

M4C: a phone with the number $\underline{6697500}$ on it

AnCMr: a phone that has the number $<$ unk $>$ on it

Ours-1: a phone that has the number 6697500 on it

Ours-2: a phone that has the word blackberry on it

Ours-3: three phones are on display including one that says " $23460 \mathrm{~g}$ "

Figure S3. More visualisation results on the TextCaps validation set. For better visualisation, the underlined word is copy from OCR tokens. The modified tokens are viewed in red colour. 


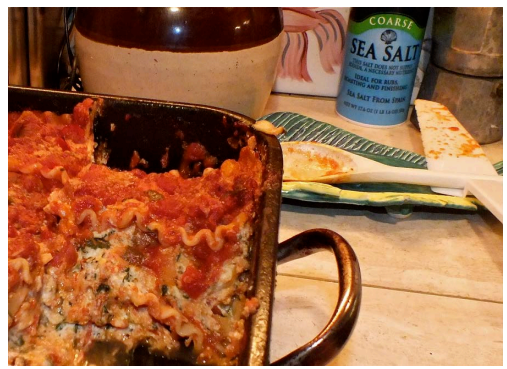

ACG: coarse sea salt from

ACMv: a bottle of <unk > <unk> sits next to a plate of food

ACMt: a bottle of sea salt is next to a plate of food

BLEU: 73.49

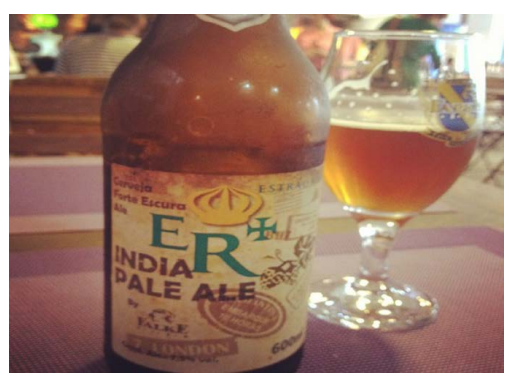

ACG: india pale ale

ACMv: a bottle of <unk><unk> ale is next to a glass of beer

ACMt: a bottle of india pale ale is next to a glass of beer

BLEU: 71.16

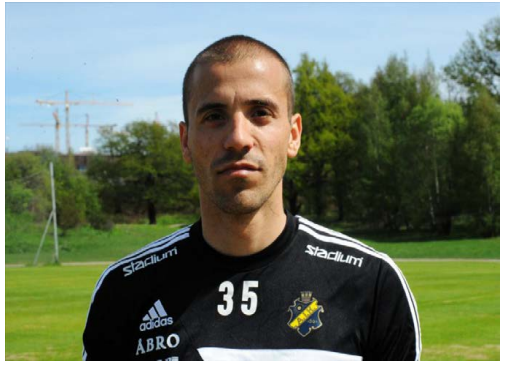

ACG: stadium 35 adidas

ACMv: a man wearing a jersey with the number 10 on it

ACMt: a man wearing a jersey with the number 35 on it

BLEU: $\mathbf{5 7 . 0 7}$

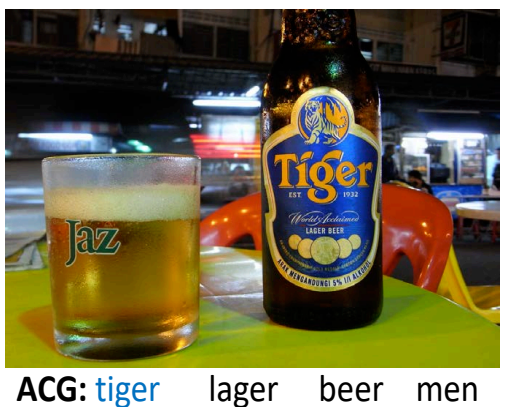

ACMv: a bottle of <unk> beer sits next to a glass of beer ACMt: a bottle of tiger beer is next to a glass of beer BLEU: 53.11

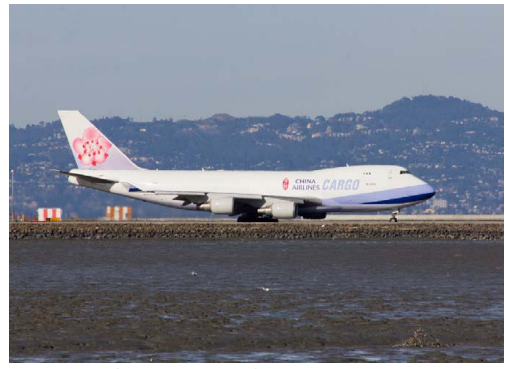

ACG: china airlines cargo ACMv: a white airlines airplane plane is on the runway ACMt: a china airlines cargo plane is on the runway BLEU: 43.17

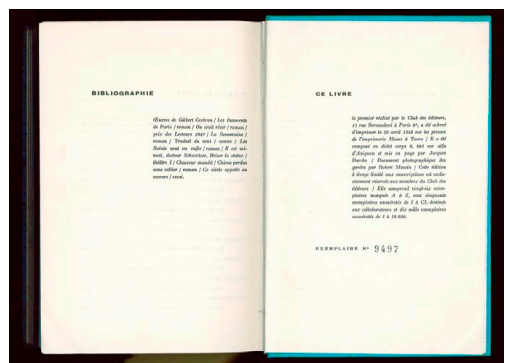

ACG: bibliographie ce exemplaire ACMv: a book is open to a page that says "'

ACMt: a book is open to a page that says bibliographie

\section{BLEU: $\mathbf{5 0 . 8 8}$}

Figure S4. Visualisation results on controllability of our method. For each image, we show the top-1 anchor-centred graph (ACG) and the generated captions of visual-captioner $\left(\mathrm{AnCM}_{v}\right)$ and text-captioner $\left(\mathrm{AnCM}_{t}\right)$. In particular, we report the BLEU score of text-captioner's output. For better visualisation, the anchor in ACG is viewed in blue colour, the underlined word is copy from ACG and the modified tokens are viewed in red colour.

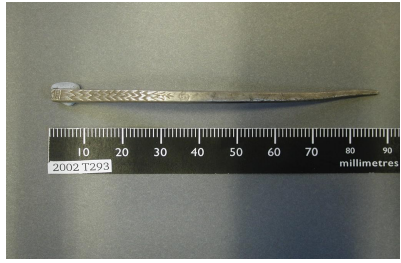

(a)

M4C: a ruler with the numbers $\underline{2002}$ on it

$\mathbf{A n C M}_{\mathbf{v}}$ : a ruler that has the number 1 through 9 on it

Ours-1: a ruler that has the numbers

1 through $\underline{\mathrm{t} 293}$ on it

Ours-2: a ruler that has the number

10 on it

Ours-3: a ruler that has the number

20 on it

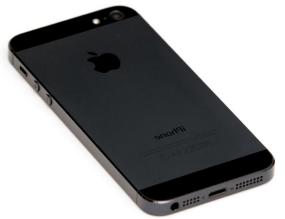

(b)
M4C: a black iphone with the back of a black background

$\mathbf{A n C M}_{\mathbf{v}}$ : a black iphone is laying face down on a white background Ours-1: a black iphone is laying face down on a white background Ours-2: a black iphone sits face down on a white surface

Ours-3: a black lg phone is laying face down on a white surface

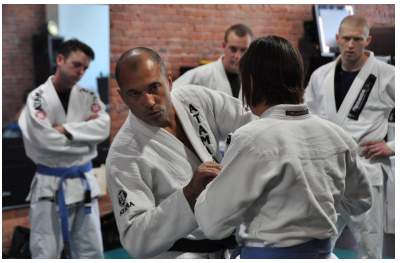

(c)

M4C: a man wearing a white shirt with the number 3 on it

AnCM $_{\mathbf{v}}$ : a group in a white shirt with the number $<$ unk $>$ on it

Ours-1: a man in a white shirt with the number 8 on it is talking to another man Ours-2: a man in a white shirt with the number 8 on it is talking to another man Ours-3: a man in a white shirt with the number 8 on it is talking to another man

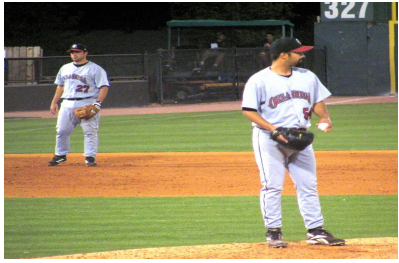

(d)

M4C: a baseball player with the number 14 on his jersey

$\mathbf{A n C M}_{\mathbf{v}}$ : a baseball player with the number 5 on his jersey

Ours-1: a baseball player with the number $\underline{321}$ on his jersey

Ours-2: a baseball player with the number 321 on his jersey

Ours-3: a baseball player with the number 321 on his jersey

Figure S5. Some failure cases of our model on the TextCaps validation set. The $<$ unk $>$ denotes 'unkown' token. The underlined word is copy from OCR tokens. The modified tokens are viewed in red colour. 